\title{
Stability Analysis of Fraction-Order Hopfield Neuron Network and Noise-Induced Coherence Resonance
}

\author{
Xuerong Shi and Zuolei Wang \\ School of Mathematics and Statistics, Yancheng Teachers University, Yancheng 224002, China \\ Correspondence should be addressed to Zuolei Wang; wzlyctc@163.com
}

Received 14 March 2020; Revised 13 May 2020; Accepted 18 May 2020; Published 29 June 2020

Academic Editor: Alessandro Lo Schiavo

Copyright (C) 2020 Xuerong Shi and Zuolei Wang. This is an open access article distributed under the Creative Commons Attribution License, which permits unrestricted use, distribution, and reproduction in any medium, provided the original work is properly cited.

In this paper, dynamical behaviors of fraction-order Hopfield neuron network are investigated. Firstly, Mittag-Leffler stability analysis is carried out and some sufficient conditions are obtained. On the basis of theoretical analysis, two criteria for determining the stability of fraction-order Hopfield neuron network are presented and comparison between them is given by theoretical analysis along with numerical simulation. According to the proposed criteria, by selecting suitable system parameters, it can be obtained that fraction-order Hopfield neuron network can stabilize to the equilibrium point or an attractor, which can be a periodic orbit or two points. Secondly, considering the inevitable noise in the complex environment of neuron network, the effect of noise on the dynamics of fraction-order Hopfield neuron network is discussed via calculating coefficient of variation and numerical simulations. Results suggest that random noise can cause coherence resonance in fraction-order Hopfield neuron network for certain noise intensity.

\section{Introduction}

In the past decades, dynamical behaviors of autonomous ordinary differential system attracted much attention. Chaotic attractor, stability, and its control have been reported in several literatures. For example, an extended Lu system was introduced, which displayed very complex dynamics for different initial conditions, such as a pair of strange attractors, a pair of limit cycles, and a pair of point attractors [1]. A novel three-dimensional chaotic system with three nonlinearities was proposed and multistability was shown for different parameters, such as one stable equilibrium, two stable equilibria and one saddle node, and two saddle foci and one saddle node [2]. An extremely simple chaotic system was constructed, which is provided with infinitely many coexisting chaotic attractors [3].

Recently, dynamical behavior of neurons attracted much attention due to its complexity and wide application in many fields. To better explore the dynamics of neurons, reliable neuron model should take the biophysical effect into account, for example, continuous exchange and pumping of calcium, sodium, and even potassium, which can induce time-varying physical magnetic in the cell. Therefore, magnetic flux [4] or field variable [5] has been included to describe the field effect. Based on the biophysical neuron models, field coupling was activated to enhance signal exchange via synchronization between neuron circuits and nonlinear circuits $[6,7]$. A 2D Hindmarsh-Rose model with a unique unstable equilibrium point was put up and multistability was observed [8]. A five-dimensional memristorcoupled neuron model was studied and coexisting phenomena of multiple firing patterns were uncovered [9]. In ordinary way, neuron is only a unit composing neuron network and its dynamics are closely related to many other neurons. Dynamics of neuron or neuron network and the application have been concerned more and more [10-14]. The multistability property was studied in impulsive multisynchronization of coupled multistable neural networks with time-varying delay [13]. Multistability and bifurcation of a delayed neural network were investigated to find a phenomenon where there coexist sixteen stable states with their own domains of attraction in the system [14]. 
Among many kinds of neuron networks, Hopfield neuron network [15] has become a prime topic thanks to its application in practical design and many results have been obtained [16-19]. A simplified Hopfield neural network (HNN) with three neurons was proposed and various behaviors were found by selecting different state initial values [16]. A novel hyperbolic-type memristor-based 3-neuron Hopfield neural network was addressed and multistable phenomena were achieved [17]. A 4D Hopfield neural network was investigated and coexistence of asymmetric self-excited attractors was exhibited [18]. A 4D autonomous Hopfield neural network was explored and multiple coexisting stable states for several sets of synaptic weight were found [19].

In the past decades, fractional calculus has been applied into various fields because of its accurate describing in many interdisciplinary fields, such as fraction-order memristive chaotic circuit [20], fractional stochastic noise [21], fractional-order relaxation-oscillation model [22], and fractional-order financial model [23]. With the expansion of application fractional calculus, dynamics of fractional-order system have been investigated. Stability and Hopf bifurcations of many fractional-order systems or networks have been discussed, such as a novel delayed fractional-order small-world network [24], a new fractional-order neural network model with multiple delays [25], a new fractionalorder chemotherapy model with two different delays [26], a class of high-dimension fractional ring-structured neural networks with multiple time delays [27], a new fractionalorder chaotic Genesio-Tesi model [28], a delayed generalized fractional-order prey-predator model [29], a new fractionalorder delayed malware propagation model [30], and fractional-order bidirectional associative memory (BAM) neural networks [31]. Delay-dependent stability and the existence of Hopf bifurcation about the order in a fractional chaotic system was proved, and threshold value of fractional-order alpha below which the chaos disappears regardless of values of system parameters was found [32].

Existing results suggested that fractional derivative is a useful tool for describing the memory property of information process. One of the advantages is that fraction order gives an extra degree of freedom for controlling system's performance. It is well known that neuron system is often in complex environment and its dynamical behaviors are inevitably influenced by biophysical effect, nonuniformal diffusion, flexible anatomic structure, and so forth. Therefore, some kinds of fraction-order neural network have been set up and dynamics of them have been explored, especially the stability analysis. A new kind of stability of fractionorder neuron network was surveyed and some criteria were obtained [33]. Stability and synchronization of fractionorder neuron network were discussed and conditions on them were presented [34, 35]. Global Mittag-Leffler stability for fraction-order neuron network with impulse effects was concerned and existence of equilibrium point was verified [36]. Multiple stability was confirmed in fraction-order neuron network with Gaussian activation function [37].

Although some results have been obtained about the dynamics of fraction-order neuron network as well as some affecting factors, analysis of the dynamical behaviors of fraction-order neuron network is still an important and challenging work. Many other factors should be further explored, such as initial values and network parameters.

In this paper, effect of network parameters on the dynamical behaviors of fraction-order Hopfield neuron network is to be investigated. Some sufficient conditions for the stability are derived and two criteria are given by theoretical analysis. Noise-induced coherence resonance of the considered network is also probed.

\section{Model Description and Preliminaries}

To analyze the dynamics of fraction-order system, several fractional calculus definitions have been proposed, among which Caputo definition [38] received great attention in many fields because it is provided with the same initial conditions as integer-order derivative. Therefore, in this paper, Caputo definition will be considered.

Definition 1 (see [38]). Suppose $\alpha>0 ; f \in C^{n+1}\left(\left[t_{0},+\right.\right.$ $\infty), R)$ is $n+1$-order continuous differentiable on $\left[t_{0},+\infty\right)$; then Caputo derivative of $\alpha$ for $f$ can be defined as

$$
{ }_{t_{0}}^{C} D_{t}^{\alpha} f(t)=\frac{1}{\Gamma(n-\alpha)} \int_{t_{0}}^{t} \frac{f^{(n)}(\tau)}{(t-\tau)^{\alpha-n+1}} \mathrm{~d} \tau,
$$

where $\Gamma(\cdot)$ is Gamma function, $t_{0}$ is positive constant, and $n-1<\alpha<n$ with positive integer $n$.

For simplicity, in the following work, Caputo's operator ${ }_{t_{0}}^{C} D_{t}^{\alpha} f(t)$ is denoted as $D^{\alpha} f(t)$.

As far as we know, integer-order Hopfield neural network (HNN) model can be represented by

$$
\dot{x}_{i}(t)=-c_{i} x_{i}(t)+\sum_{j=1}^{n} a_{i j} f_{j}\left(x_{j}(t)\right)+I_{i}, \quad i=1,2, \ldots, n, t>0,
$$

which is a system of differential equations. In (2), $x(t)=$ $\left(x_{1}(t), x_{2}(t), \ldots, x_{n}(t)\right)^{T}$ is state variable, $f_{j}(\cdot)$ is activation function of the $j$ th neuron, $a_{i j}$ is a constant indicating synaptic connection weight between the $i$ th neuron and the $j$ th neuron, and positive constant $c_{i}$ and $I_{i}$ are self-regulating parameter and external signal of the $i$ th neuron, respectively.

Fractional-order neural network has received much attention in control, intelligent processing, and other fields due to its unique structure of knowledge representation and the method of information processing. Therefore, starting from (2), replace the usual integer-order derivative by the fractional-order Caputo derivative, and then fraction-order Hopfield neuron network (FHNN) can be obtained as

$$
D^{\alpha} x_{i}(t)=-c_{i} x_{i}(t)+\sum_{j=1}^{n} a_{i j} f_{j}\left(x_{j}(t)\right)+I_{i}, \quad i=1,2, \ldots, n, t>0,
$$

which is composed of $n$ neurons. In (3), $\alpha \in(0,1)$ and the explanations of other parameters are the same as those in (2). 
According to [39], Lipschitz-continuous function $f_{j}$ can make equation have a unique solution. To gain the main results of this paper, Assumption 1 is given.

Assumption 1. It is assumed that the neuron activation function $f_{j}$ in (3) is Lipschitz-continuous on $R$; that is,

$$
\left|f_{j}(u)-f_{j}(v)\right| \leq L_{j}|u-v|,
$$

holds for all $u, v \in R(u \neq v)$ and $f_{j}(0)=0,(j=1,2, \ldots, n)$, where positive $L_{j}$ is Lipschitz-constant.

When solving the problem of fractional differential equation, Mittag-Leffler function is often used, which plays the same role as the exponential function for solving integerorder differential equation and can be defined as

$$
E_{\alpha, \beta}(z)=\sum_{k=0}^{\infty} \frac{z^{k}}{\Gamma(k \alpha+\beta)},
$$

where $\alpha>0, \beta>0$ and $z \in C$. For $\beta=1$, formula (6) can be simplified as

$$
E_{\alpha}(z)=\sum_{k=0}^{\infty} \frac{z^{k}}{\Gamma(k \alpha+1)}
$$

which involves only one parameter $\alpha$. From (5) and (6), it can be known that $E_{\alpha}(z)=E_{\alpha, 1}(z)$; particularly, $E_{1,1}(z)=e^{z}$.

Furthermore, Laplace transform of Mittag-Leffler function (5) can be obtained as

$$
\mathscr{L}\left\{t^{\beta-1} E_{\alpha, \beta}\left(-\lambda t^{\alpha}\right)\right\}=\frac{s^{\alpha-\beta}}{s^{\alpha}+\lambda},
$$

where $t$ and $s$ are variables from time domain and Laplace domain, respectively. $s$ is satisfied with the condition $\operatorname{Re}(s)>\left|\lambda^{1 / \alpha}\right|$.

When the equilibrium point $\bar{x}=0$, definition of MittagLeffler stability of fraction-order system was given in [40]. If the equilibrium point $x^{*} \neq 0$, via transformation, we can get corresponding result as depicted in Definition 2.

Definition 2. For the equilibrium point $x^{*}\left(x^{*} \neq 0\right)$ of FHNN (3), if there exists constant $\lambda>0$, and for any solution $x(t)$ of FHNN (3) with initial value $x_{0}$, when $t \geq 0$,

$$
\left\|x(t)-x^{*}\right\| \leq\left\|x_{0}-x^{*}\right\| E_{\alpha}\left(-\lambda t^{\alpha}\right),
$$

can be obtained; then it is said that the solution is globally Mittag-Leffler-stable at $x^{*}$.

According to the result in [41], global Mittag-Leffler stability means globally asymptotic stability.

\section{Stability Analysis}

In this section, stability of FHNN (3) is discussed via theoretical analysis and numerical simulations.

3.1. Theoretical Analysis. According to the preliminaries in Section 2, stability of FHNN (3) is to be analyzed and some sufficient conditions are to be derived. For this end, two lemmas are introduced firstly.
Lemma 1 (see [42]). It is assumed that $\alpha \in(0,1), \lambda$ is a positive constant, and $V(t)$ is continuous on $[0,+\infty)$ satisfying

$$
D^{\alpha} V(t) \leq-\lambda V(t)
$$

and then, for $t \geq 0$, we have

$$
V(t) \leq V(0) E_{\alpha}\left(-\lambda t^{\alpha}\right) .
$$

Lemma 2 (see [38]). For continuous differentiable function $h(t) \in C^{1}([0,+\infty), R)$, the inequality

$$
{ }_{0} D_{t}^{\alpha}|h(t)| \leq \operatorname{sgn}(h(t))_{0} D_{t}^{\alpha} h(t), \quad 0<\alpha<1,
$$

holds almost everywhere.

Existence of equilibrium points is given as follows in Theorem 1.

Theorem 1. Considering FHNN (3), if Assumption 1 holds, constants $a_{i j}$ and $c_{i}$ satisfy

$$
c_{i}-\sum_{j=1}^{n}\left|a_{j i}\right| L_{i}>0
$$

and then there is unique equilibrium point $x^{*}$ of FHNN (3).

Proof. Suppose that $H(u)=\left(H_{1}(u), H_{2}(u), \ldots, H_{n}(u)\right)^{T}$ with

$$
H_{i}(u)=\sum_{j=1}^{n} a_{i j} f_{j}\left(\frac{u_{j}}{c_{j}}\right)+I_{i}, \quad i, j=1,2 \ldots, n,
$$

where $u=\left(u_{1}, u_{2}, \ldots, u_{n}\right)^{T}$ and $f_{j}$ is Lipschitz-continuous function with Lipschitz-constant $L_{j}>0$. Then, for any vectors $u=\left(u_{1}, u_{2}, \ldots, u_{n}\right)^{T} \in R^{n}$ and $v=\left(v_{1}, v_{2}, \ldots, v_{n}\right)^{T} \in R^{n}$, we can obtain

$$
\left|H_{i}(u)-H_{i}(v)\right|=\left|\sum_{j=1}^{n} a_{i j}\left[f_{j}\left(\frac{u_{j}}{c_{j}}\right)-f_{j}\left(\frac{v_{j}}{c_{j}}\right)\right]\right| \leq \sum_{j=1}^{n} \frac{L_{j}}{c_{j}}\left|a_{i j}\right|\left|u_{j}-v_{j}\right| .
$$

Sum both sides of (14), and $\sum_{i=1}^{n}\left|H_{i}(u)-H_{i}(v)\right| \leq$ $\sum_{i=1}^{n} \sum_{j=1}^{n}\left(L_{j} / c_{j}\right)\left|a_{i j}\right|\left|u_{j}-v_{j}\right|$ can be derived.

According to (12), $\left(L_{i} / c_{i}\right) \sum_{j=1}^{n}\left|a_{j i}\right|<1$ can be obtained. For simplicity, let

$$
\theta=\frac{L_{i}}{c_{i}} \sum_{j=1}^{n}\left|a_{j i}\right|
$$

and then we can get

$$
\sum_{i=1}^{n}\left|H_{i}(u)-H_{i}(v)\right| \leq \theta \sum_{i=1}^{n}\left|u_{j}-v_{j}\right| .
$$

From (16), it is easy to know that $\left|H_{i}(u)-H_{i}(v)\right| \leq \theta\left|u_{j}-v_{j}\right|$, while $\left|u_{j}-v_{j}\right|<|u-v|$. Then, $H: R^{n} \longrightarrow R^{n}$ can be regarded as a contraction mapping on $R^{n}$. In light of the principle of compression mapping, FHNN (3) is provided with a unique equilibrium point $x^{*}=\left(x_{1}, x_{2}, \ldots, x_{n}\right)^{T}$. The proof is completed. 
When $x^{*}=0$, it is easy to gain the stability of FHNN (3) at equilibrium point $x^{*}$. When $x^{*} \neq 0$, via transformation

$$
v_{i}(t)=x_{i}(t)-x_{i}^{*}(t), \quad i=1,2, \ldots, n,
$$

$x^{*}$ can be shifted to the origin and FHNN (3) can be transformed into

$$
D^{\alpha} v_{i}(t)=-c_{i} v_{i}(t)+\sum_{j=1}^{n} a_{i j} g_{j}\left(v_{j}(t)\right),
$$

where $g_{j}\left(v_{j}(t)\right)=f_{j}\left(v_{j}(t)+x_{j}^{*}\right)-f_{j}\left(x_{j}^{*}\right)$.

According to the above, some results about the stability of FHNN (3) are depicted as in the following theorems.

Theorem 2. If Assumption 1 holds, constants $a_{i j}$ and $c_{i}$ in network (3) satisfy

$$
\lambda=\min _{1 \leq i \leq n}\left\{c_{i}-L_{i} \sum_{j=1}^{n}\left|a_{j i}\right|\right\}>0,
$$

and then FHNN (3) is globally Mittag-Leffler-stable and solution of FHNN (3) will stabilize to the unique equilibrium point $x^{*}$.

Remark 1. Formula (19) suggests that $c_{i}-L_{i} \sum_{j=1}^{n}\left|a_{j i}\right|,(i=$ $1,2,3, \ldots, n)$ are all positive, from which it is known that formula (12) is true. That is to say, condition (19) ensures the existence and of uniqueness of the equilibrium point of FHNN (3).

Proof. In terms of condition (19), it can be known that FHNN (3) has unique equilibrium point. Suppose $x^{*}$ is unique equilibrium point and construct Lyapunov function

$$
V(t)=\sum_{i=1}^{n}\left|v_{i}(t)\right|
$$

and, on the basis of Lemma 2, $v_{i}(t)$ satisfies

$$
{ }_{0} D_{t}^{\alpha}\left|v_{i}(t)\right| \leq \operatorname{sgn}\left(v_{i}(t)\right)_{0} D_{t}^{\alpha} v_{i}(t)
$$

According to formula (19), $\lambda=\min \left\{c_{i}-L_{i} \sum_{j=1}^{n} a_{j i}\right\}>0$ holds. Fraction-order derivative of $V(t)$ along FHNN (3) can be derived as

$$
\begin{aligned}
D^{\alpha} V(t) & =\sum_{i=1}^{n} D^{\alpha}\left|v_{i}(t)\right| \\
& \leq \sum_{i=1}^{n} \operatorname{sgn}\left(v_{i}(t)\right)\left\{-c_{i} v_{i}(t)+\sum_{j=1}^{n} a_{j i} g_{j}\left(v_{j}(t)\right)\right\} \\
& \leq \sum_{i=1}^{n}\left\{-c_{i}\left|v_{i}(t)\right|+\sum_{j=1}^{n}\left|a_{j i}\right| L_{j}\left|v_{j}(t)\right|\right\} \\
& =-\sum_{i=1}^{n}\left\{c_{i}-\sum_{j=1}^{n}\left|a_{j i}\right| L_{i}\right\}\left|v_{i}(t)\right| \\
& \leq-\lambda V(t) .
\end{aligned}
$$

According to Lemma 1, one has

$$
V(t) \leq V(0) E_{\alpha}\left(-\lambda t^{\alpha}\right)
$$

which implies that

$$
\sum_{i=1}^{n}\left|x_{i}(t)-x_{i}^{*}\right| \leq\left(\sum_{i=1}^{n}\left|x_{i}(0)-x_{i}^{*}\right|\right) E_{\alpha}\left(-\lambda t^{\alpha}\right) .
$$

It means that

$$
\left\|x(t)-x^{*}\right\| \leq\left\|x_{0}-x^{*}\right\| E_{\alpha}\left(-\lambda t^{\alpha}\right), \quad t \geq 0 .
$$

Then one has

$$
\left\|x(t)-x^{*}\right\| \longrightarrow 0, \quad t \longrightarrow+\infty .
$$

It follows that $x^{*}$ is uniformly attractive, which implies that FHNN (3) achieves global Mittag-Leffler stability at the unique equilibrium point $x^{*}$. Theorem 2 is proved.

In the proof of Theorem 2, Lyapunov function is constructed by using the absolute value function. Compared with general Lyapunov function, the addressed Lyapunov function has certain advantage. On the one hand, it is simple for calculation; on the other hand, by introducing sign function, the discussion of nonsmoothness can be avoided.

Theorem 3. If Assumption 1 holds, constants $a_{i j}$ and $c_{i}$ in FHNN (3) satisfy

$$
\lambda=\underset{1 \leq i \leq n}{\forall}\left\{c_{i}-L_{i} \sum_{j=1}^{n} a_{j i}\right\}>0,
$$

and then FHNN (3) is globally Mittag-Leffler-stable.

Remark 2. Formula (27) means that there exists one positive value in all $c_{i}-L_{i} \sum_{j=1}^{n} a_{j i}(i=1,2, \ldots n)$, which is less conservative compared with condition (19) in Theorem 2. Theorem 3 can extend the range of $\lambda$ and can be received in more applications. Furthermore, Theorem 3 need not require $\theta<1$ for system parameters and may not ensure that equilibrium point of FHNN (3) is unique. In other words, under the condition of (27) in Theorem 3, we only can know the global Mittag-Leffler stability. FHNN (3) can be globally Mittag-Leffler-stable at equilibrium point $x^{*}$ or attraction domain around $x^{*}$.

Remark 3. The proof of Theorem 3 is analogous to that of Theorem 2 through applying fractional-order Lyapunov direct method. So the detailed proof of Theorem 3 is omitted here. It should be noted that, in the proof of Theorems 2 and 3 , existence of positive $c_{i}-L_{i} \sum_{j=1}^{n} a_{j i}$ is essential, which can be achieved under the condition in both Theorems 2 and 3 . Theorem 2 suggests that all $c_{i}-L_{i} \sum_{j=1}^{n} a_{j i}$ are positive, while Theorem 3 can ensure the existence of positive $c_{i}-L_{i} \sum_{j=1}^{n} a_{j i}$. Namely, Theorem 2 is a special case of Theorem 3. Therefore, the proof of Theorem 3 can be obtained by replacing $\lambda$ with certain positive $\lambda_{i}$ in formula (22).

3.2. Numerical Simulations. In the simulations to verify Theorems 2 and 3, MATLAB program and Adams- 
Bashforth-Moulton predictor-corrector algorithm [43] are implemented and the initial conditions $x_{i}(t)(i=1,2,3)$ are considered as Gaussian white noise.

3.2.1. Simulations about Theorem 2. The system parameters are selected as $c_{1}=c_{2}=c_{3}=3, I_{1}=0.5, I_{2}=1.2$, and $I_{3}=$ -0.8 and the matrix of synaptic connection weight is taken as

$$
\left(a_{i j}\right)=\left(\begin{array}{ccc}
-1.2 & 0.3 & -1.5 \\
-0.9 & 1 & -0.8 \\
0.5 & -1.2 & 0.6
\end{array}\right)
$$

The activation functions are taken as $f_{j}\left(x_{j}\right)=\tanh \left(x_{j}\right)(j=1,2,3)$. By calculation, we know that conditions in Theorem 2 are satisfied for $L_{i}=1(i=1,2,3)$, $\lambda=0.1$, and $\theta<1$. According to Theorem 2, unique equilibrium point of FHNN (3) can be calculated as $x^{*}=(0.33,0.61,-0.53)^{T}$ and FHNN (3) is globally MittagLeffler-stable at $x^{*}$. The time revolutions of state variables $x_{i}(t)(i=1,2,3)$ with $\alpha=0.95$ and $\alpha=0.55$ are calculated and depicted in Figures 1 and 2, respectively.

From Figures 1 and 2, it can be known that solution of FHNN (3) is stable at the equilibrium point $x^{*}=(0.33,0.61,-0.53)^{T}$ for different value of $\alpha$, which means that, in this case, solution of FHNN (3) is stable equilibrium point. That is to say, the order of FHNN (3) has little effect on the convergence essence of the solution. But, via further observation, it is found that the value of $\alpha$ affects the convergence speed. The smaller $\alpha$ is, the less the time for converging to the equilibrium point is.

3.2.2. Simulations about Theorem 3. For comparison with Theorem 2, in following simulations, the activation functions are also chosen as $f_{j}\left(x_{j}\right)=\tanh \left(x_{j}\right)(j=1,2,3)$. Three different cases are taken into account.

Case 1. Take $\alpha=0.8, \quad I_{1}=0.5, \quad I_{2}=1.2, \quad I_{3}=-0.8$, $c_{1}=6.0468, c_{2}=1.2361$, and $c_{3}=1.2939$ and

$$
\left(a_{i j}\right)=\left(\begin{array}{ccc}
-3.6251 & 1.7318 & 4.9492 \\
-5.3548 & -1.4211 & -0.2666 \\
-6.2992 & 2.6603 & -1.3113
\end{array}\right) \text {, }
$$

and it can be calculated that $\lambda=\min _{1 \leq i \leq n}\left\{c_{i}-L_{i} \sum_{j=1}^{n} \mid\right.$ $\left.a_{j i} \mid\right\}=-9.2323<0$, which suggests that Theorem 2 is invalid for this case. But there exists $c_{1}-L_{1} \sum_{j=1}^{n} a_{j 1}=21.3259>0$; according to Theorem 3, we can assert the Mittag-Leffler stability of FHNN (3). The dynamical behaviors of FHNN (3) are shown in Figure 3, which shows that FHNN (3) gradually converges to equilibrium point $x^{*}=(0.06,0.35,-0.30)^{T}$; namely, $x^{*}=(0.06,0.35,-0.30)^{T}$ is a stable equilibrium point.

Figure 3 indicates that FHNN (3) still tends to the equilibrium point over time $t$ under the condition of only $c_{1}-L_{1} \sum_{j=1}^{n} a_{j 1}=21.3259>0$, which means it remains globally Mittag-Leffler-stable.
Case 2. Choose $\alpha=0.8, I_{1}=0.5, I_{2}=1.2, I_{3}=-0.8, c_{1}=3$, $c_{2}=3$, and $c_{3}=3$ and

$$
\left(a_{i j}\right)=\left(\begin{array}{ccc}
0.5809 & -1.5057 & 2.7260 \\
-4.3061 & 1.4389 & -2.6839 \\
-1.0590 & 1.7481 & 3.2859
\end{array}\right),
$$

and calculation results suggest $c_{i}-L_{i} \sum_{j=1}^{n} a_{j i}>0(i=1,2)$; $\lambda=\min _{1 \leq i \leq n}\left\{c_{i}-L_{i} \sum_{j=1}^{n}\left|a_{j i}\right|\right\}=-5.6958<0$. It means that the parameters in this case still only satisfy Theorem 3 . The dynamical behaviors of FHNN (3) in this case are depicted in Figure 4, which indicates that the solution of FHNN (3) converges to a limit cycle, which is a periodic oscillation in the neighborhood of equilibrium point $x^{*}=(0.03,0.50,0.1)^{T}$ and can be regarded as the attractor of FHNN (3).

Case 3. Choose $\alpha=0.8, I_{1}=0.5, I_{2}=1.2, I_{3}=-0.8, c_{1}=3$, $c_{2}=3$, and $c_{3}=3$ and

$$
\left(a_{i j}\right)=\left(\begin{array}{ccc}
9.3258 & -1.9924 & -0.1482 \\
8.4845 & -3.6262 & 1.7487 \\
-3.4041 & 3.2592 & 1.0357
\end{array}\right) \text {, }
$$

and, by calculation, it can be known that only Theorem 3 is satisfied. The dynamical behaviors of FHNN (3) are drawn in Figure 5, which illustrates that FHNN (3) exhibits bistable state, which can be regarded as a brand-new and important dynamical form of the neural network. The generation of the bistability is dependent on initial values. In this case, although FHNN (3) has only one equilibrium point, it can have two domains of attraction corresponding to initial values. The solution of FHNN (3) tends to two points accidently, which can be regarded as the attractor of FHNN (3).

Remark 4. Compared with publications that deal with the Mittag-Leffler stability, a phenomenon is found that, for suitable parameters, although FHNN (3) only has one equilibrium point, it can show multiple stabilities.

From Figures 3-5, it is easy to know that effect of system parameters is critical in characterizing neuron network's dynamics.

\section{Noise-Induced Coherence Resonance}

Via stability analysis, it can be obtained that FHNN (3) can reach global Mittag-Leffler stability for suitable system parameters. But, as we all know, neural network is inevitably disturbed by noise, which can destroy the stability and lead to coherence resonance. As a kind of inevitable noise, the electromagnetic induction may appear in the complex environment of neuron network. To obtain the effects of electromagnetic induction on the dynamics of neuron network, dynamics analysis of memristor synapse-coupled Hopfield neural network has been executed and results suggested that memristive Hopfield neural network can show the coexistence of chaotic and stable point attractors [44], coexistence of multistable patterns of the spiral chaotic 


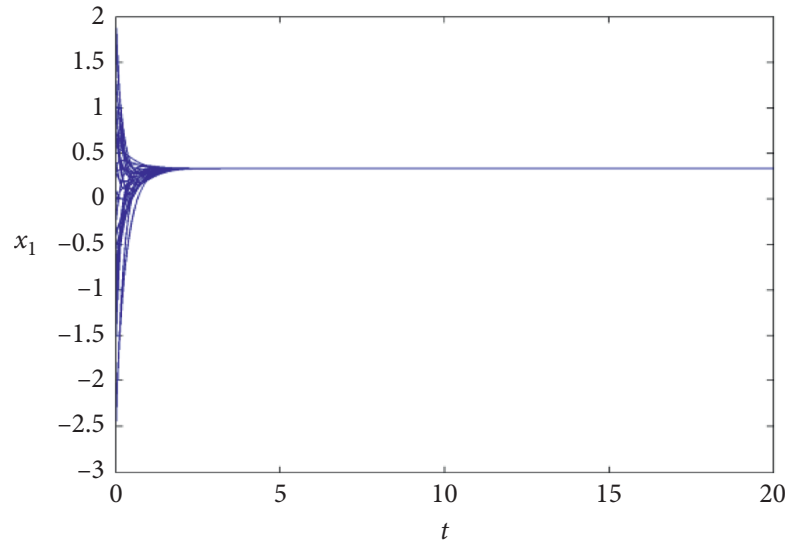

(a)

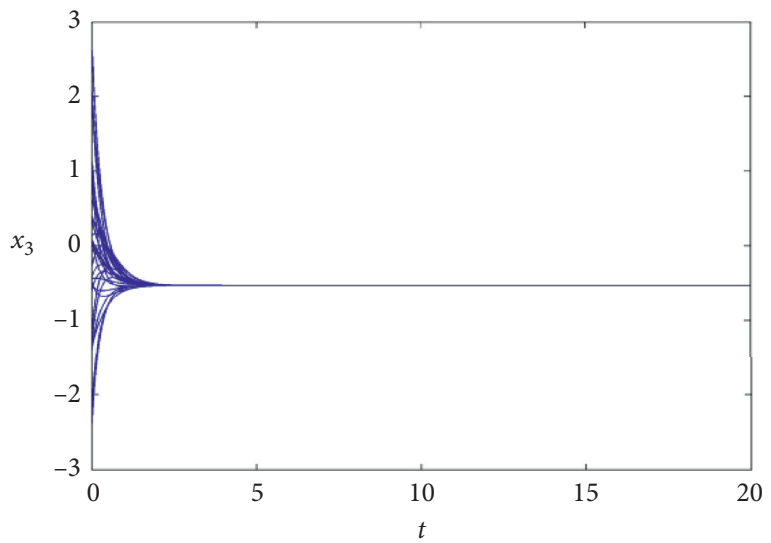

(c)

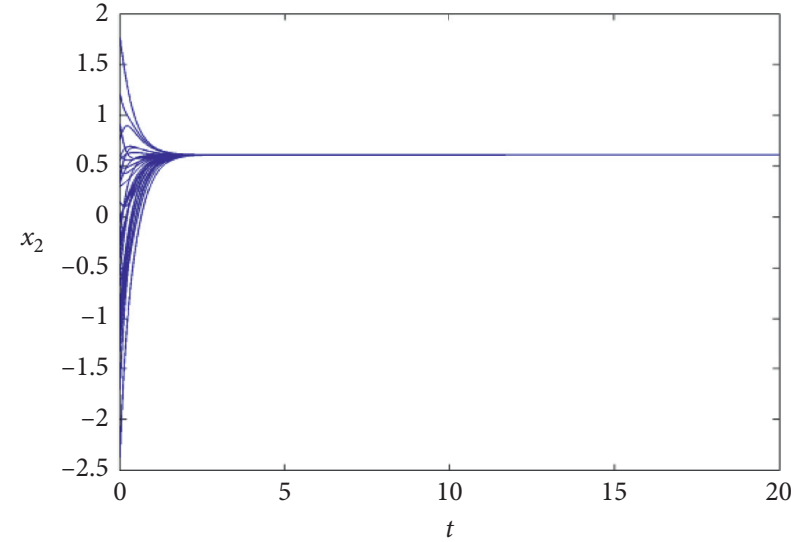

(b)

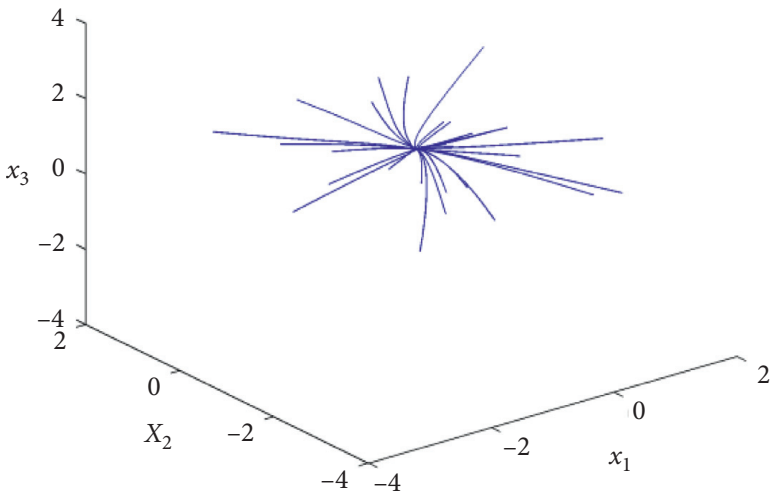

(d)

Figure 1: Mittag-Leffler stability of FHNN (3) with parameters satisfying (19) and $\alpha=0.95$.

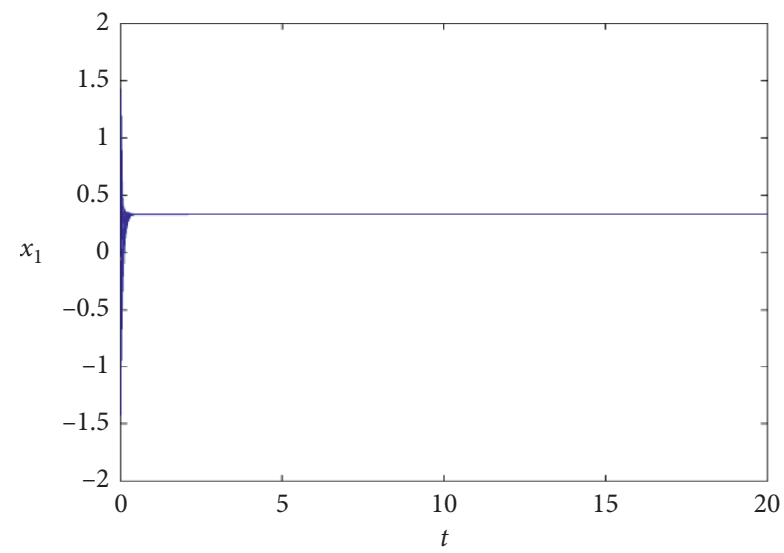

(a)

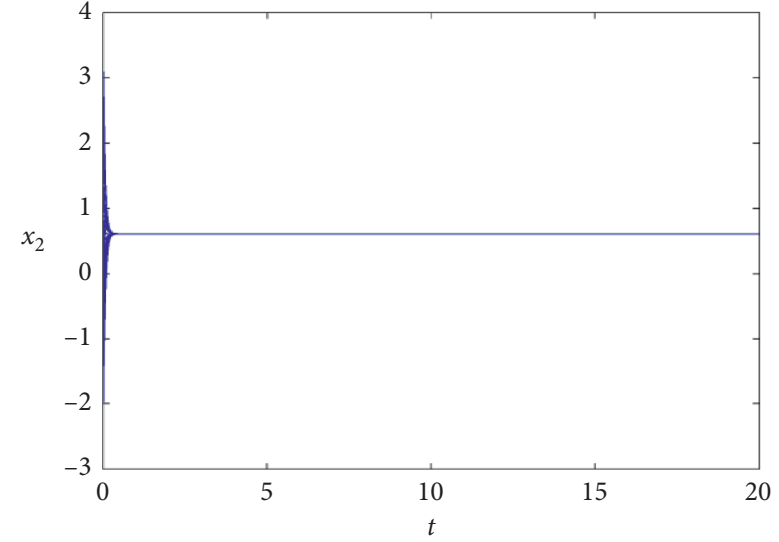

(b)

Figure 2: Continued. 


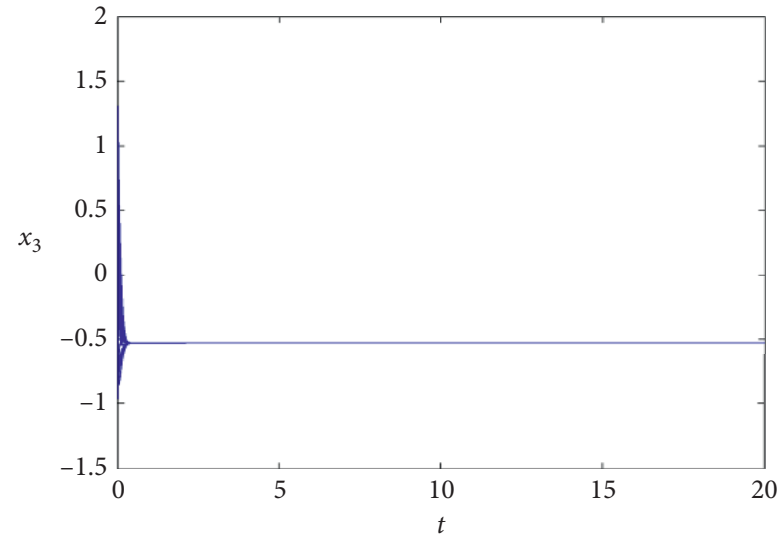

(c)

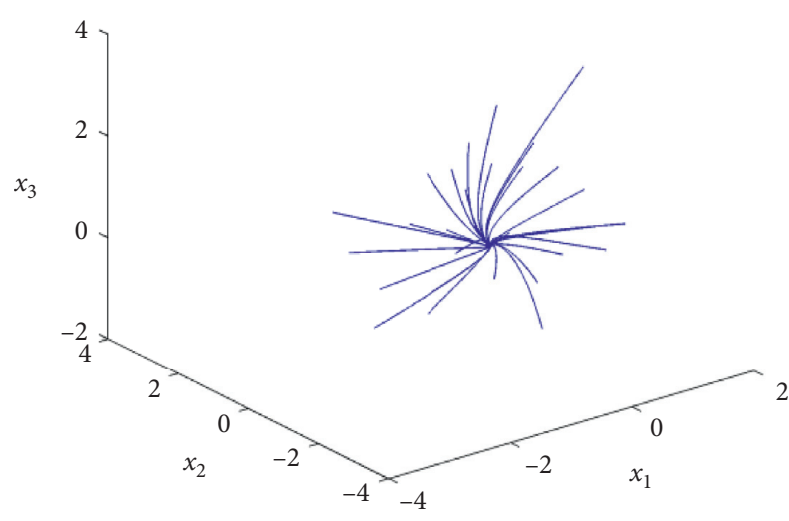

(d)

Figure 2: Mittag-Leffler stability of FHNN (3) with parameters satisfying (19) and $\alpha=0.55$.

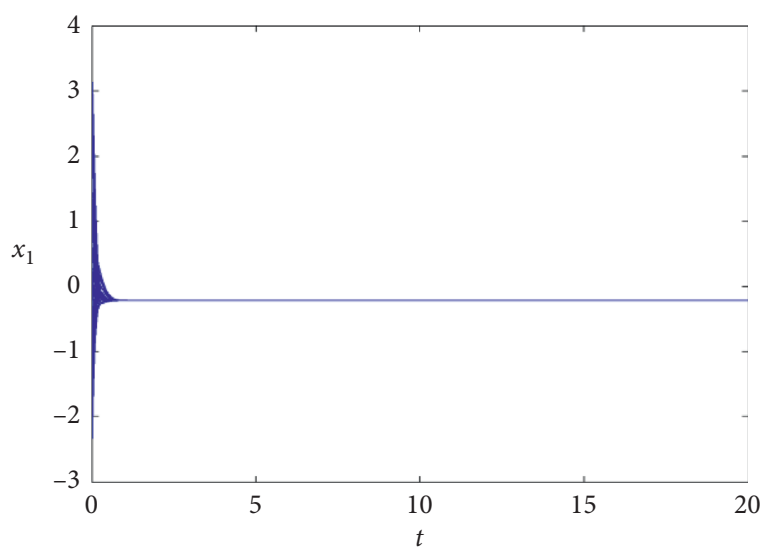

(a)

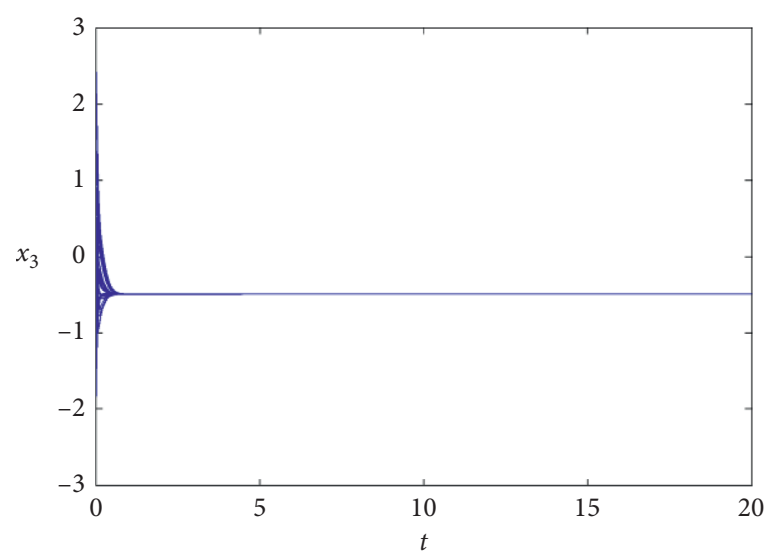

(c)

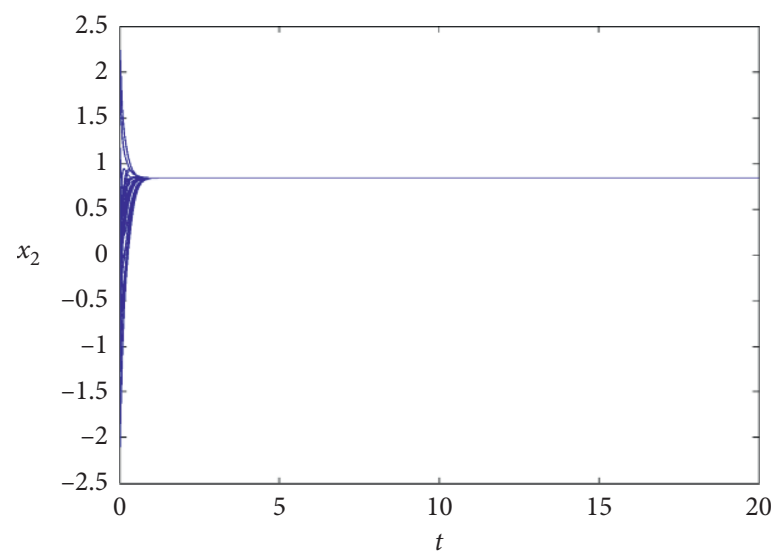

(b)

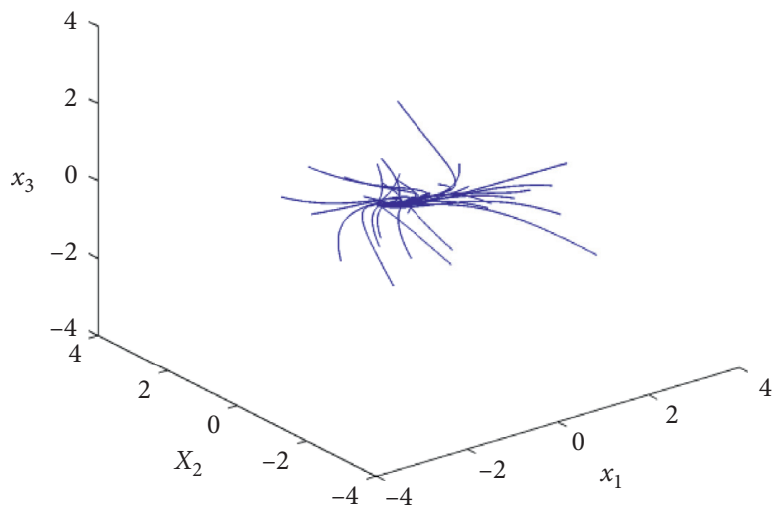

(d)

Figure 3: Evolution of FHNN (3) with parameters satisfying (27) and $\alpha=0.8$ in Case 1.

patterns, and stable resting patterns [45]. In this paper, the effect of Gaussian white noise on the dynamics of FHNN (3) is discussed. It is supposed that the first neuron is disturbed by noise. Then the considered network can be written as

$$
\left\{\begin{array}{l}
D^{\alpha} x_{1}(t)=-c_{1} x_{1}(t)+\sum_{j=1}^{n} a_{1 j} f_{j}\left(x_{j}(t)\right)+I_{1}+\xi(t), \\
D^{\alpha} x_{i}(t)=-c_{i} x_{i}(t)+\sum_{j=1}^{n} a_{i j} f_{j}\left(x_{j}(t)\right)+I_{i}, \quad i=2, \ldots, n,
\end{array}\right.
$$




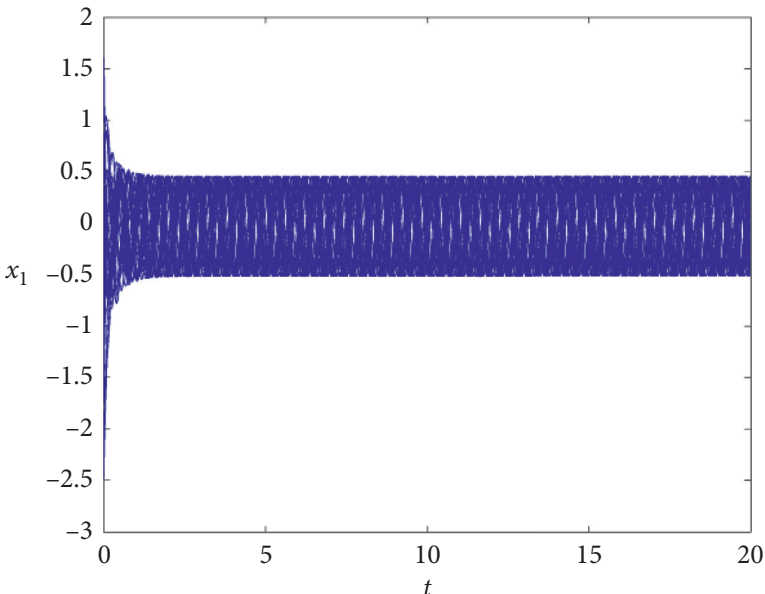

(a)

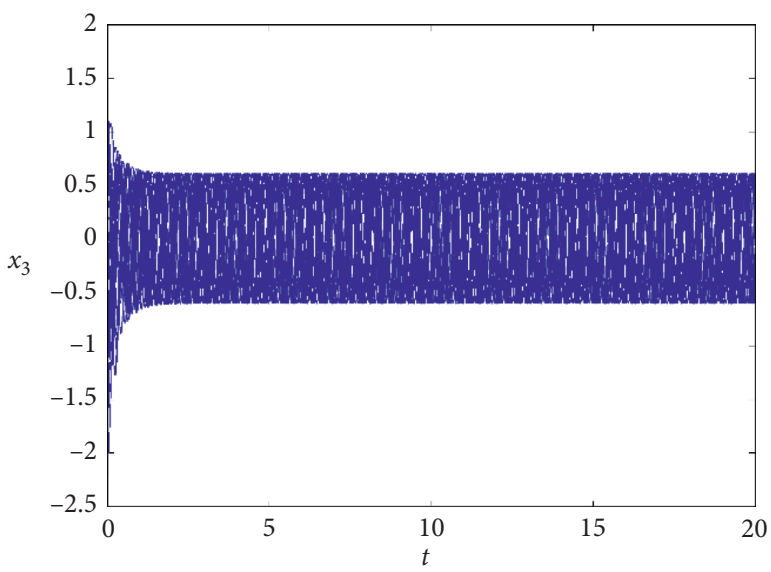

(c)

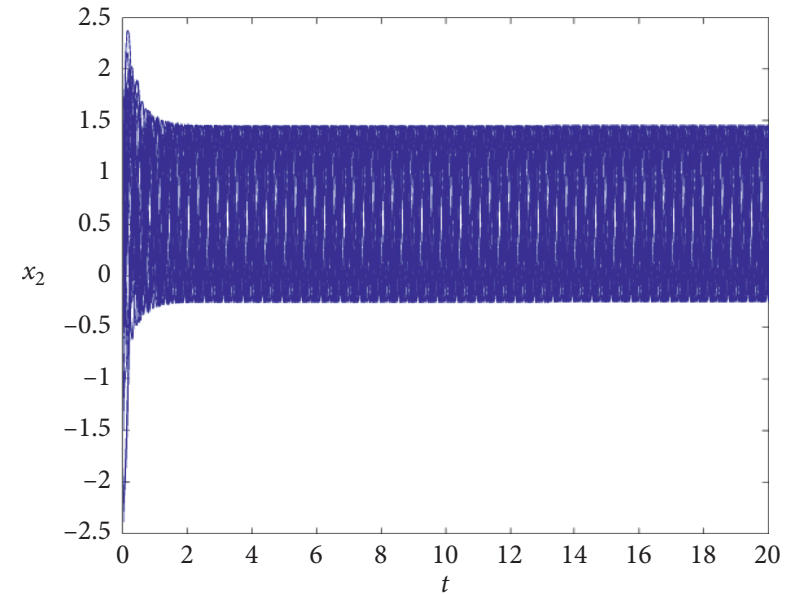

(b)

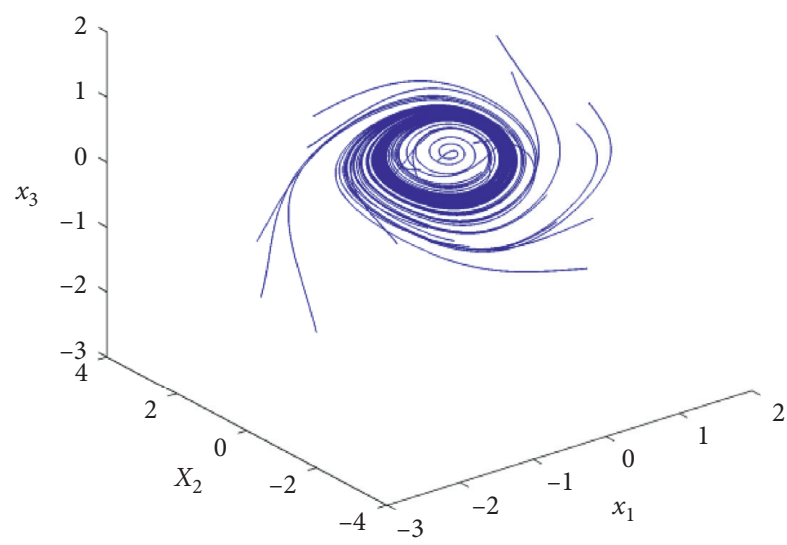

(d)

Figure 4: Evolution of FHNN (3) with parameters satisfying (27) and $\alpha=0.8$ in Case 2.

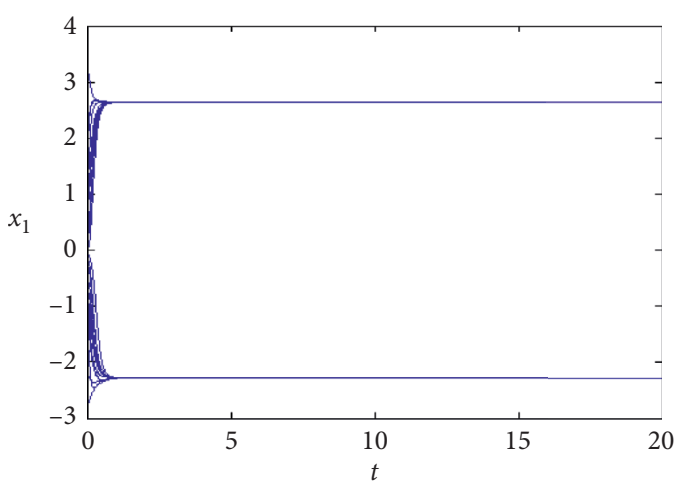

(a)

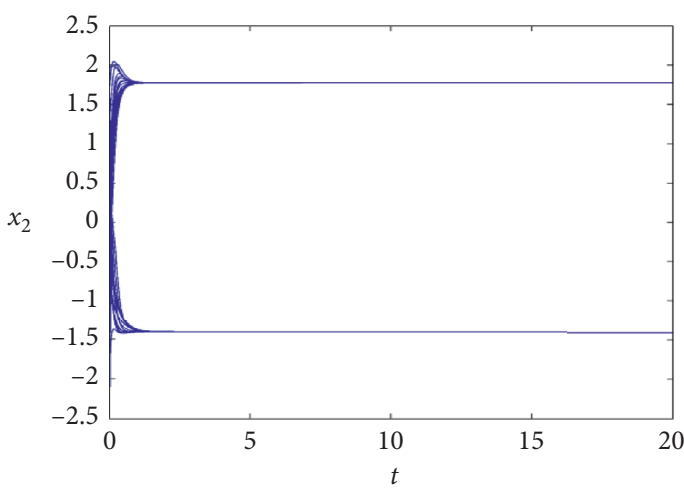

(b)

FIgURE 5: Continued. 


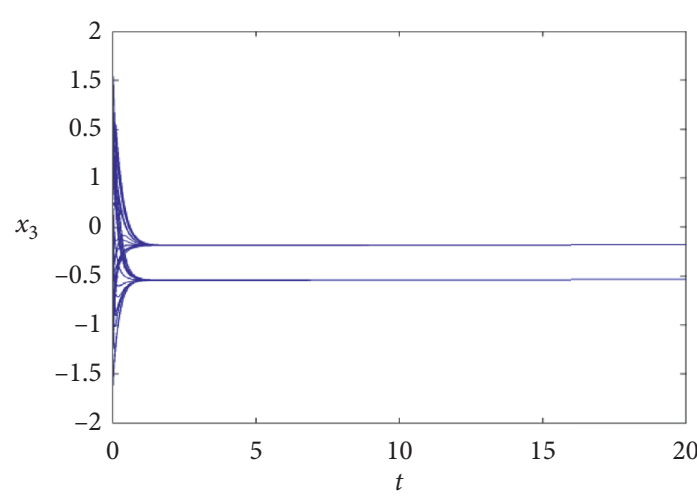

(c)

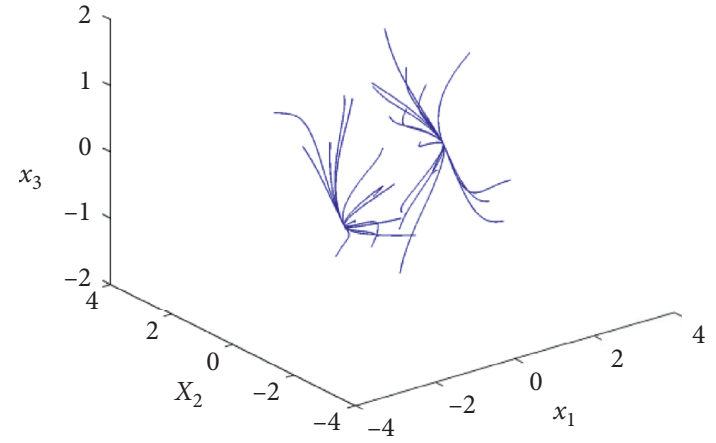

(d)

FIGURE 5: Bistability of FHNN (3) dependent on the initial values.

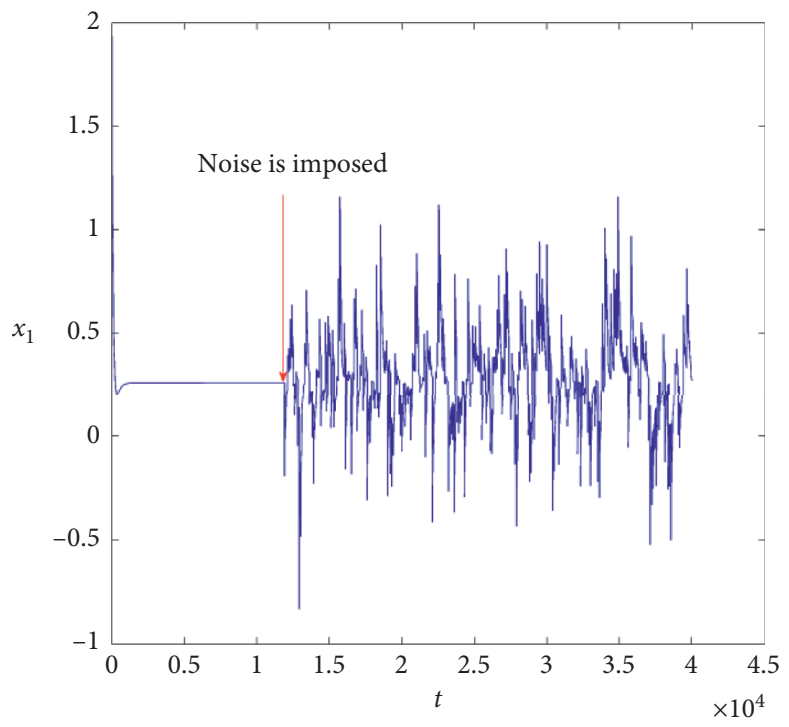

(a)

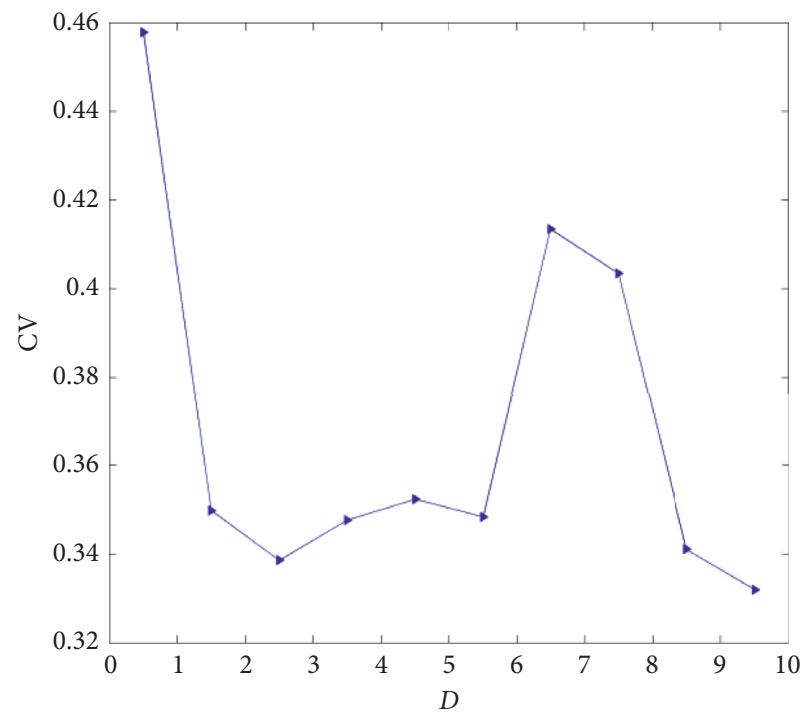

(b)

FIgURE 6: Time series of $x_{1}$ in FHNN (32) disturbed by noise and CV trend with noise intensity changing when parameters satisfy Theorem 2 for $\alpha=0.55$. (a) Time series of $x_{1}$; (b) CV trend with noise intensity $D$ changing.

where $\xi(t)$ is Gaussian white noise with $\xi(t)=0, \xi(t) \xi\left(t^{\prime}\right)=$ $2 D \delta\left(t-t^{\prime}\right)$ and $D$ is noise intensity. To investigate the coherence resonance of FHNN (32) induced by noise, as an irregularity index of the neuron network, coefficient of variation is introduced as

$$
\mathrm{CV}=\frac{\sqrt{\left\langle\mathrm{ISI}^{2}\right\rangle-\langle\mathrm{ISI}\rangle^{2}}}{\langle\mathrm{ISI}\rangle},
$$

which is the ratio of the standard deviation of ISI to the mean of ISI. The smaller the CV is, the more regular the network presents, which means that the network is provided with better synchronization. The larger the $\mathrm{CV}$ is, the more irregular the network appears.

To discuss the coherence resonance of FHNN (32) by noise, noise is added when time series develops to a certain time, as an illustration, at $t=12000$ time units. Dynamics of FHNN (32) are calculated and depicted in Figures 6-9, which reveal the noise-induced coherence resonance in FHNN (32). The parameters in Figures 6 and 7 are taken to be the same as those in Figures 2 and 3, respectively. Figures 6 and 7 indicate that Gaussian white noise can excite FHNN (32) from stability to vibration and produce coherence resonance. The parameters in Figure 8 are chosen the same as those in Figure 4. Figure 8 suggests that Gaussian white noise can suppress the dynamical behaviors of FHNN (32) and make it change from periodic state to chaotic state. The variation of noise intensity also can induce coherence resonance. The parameters in Figure 9 are the same as those in Figure 5. Figure 9 confirmed that random noise can also make bistable FHNN [3] produce coherence resonance. 


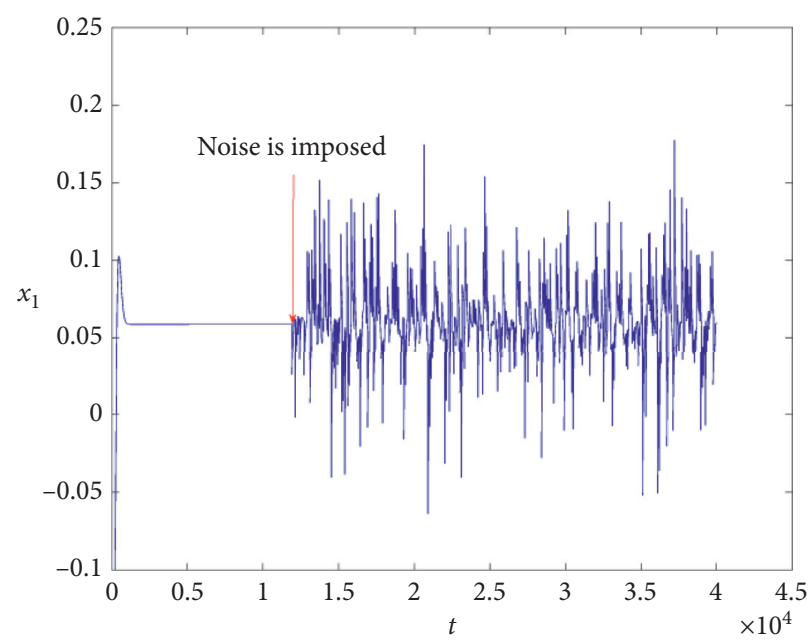

(a)

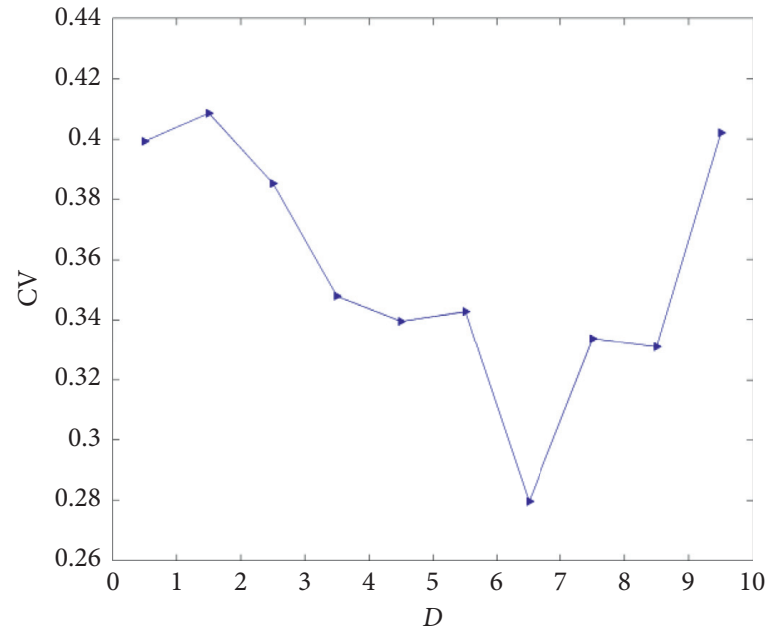

(b)

FIgURE 7: Time series of $x_{1}$ in FHNN (32) disturbed by noise and CV trend with noise intensity changing when parameters satisfy Theorem 3 in Case 1 for $\alpha=0.8$. (a) Time series of $x_{1}$; (b) CV trend with noise intensity $D$ changing.

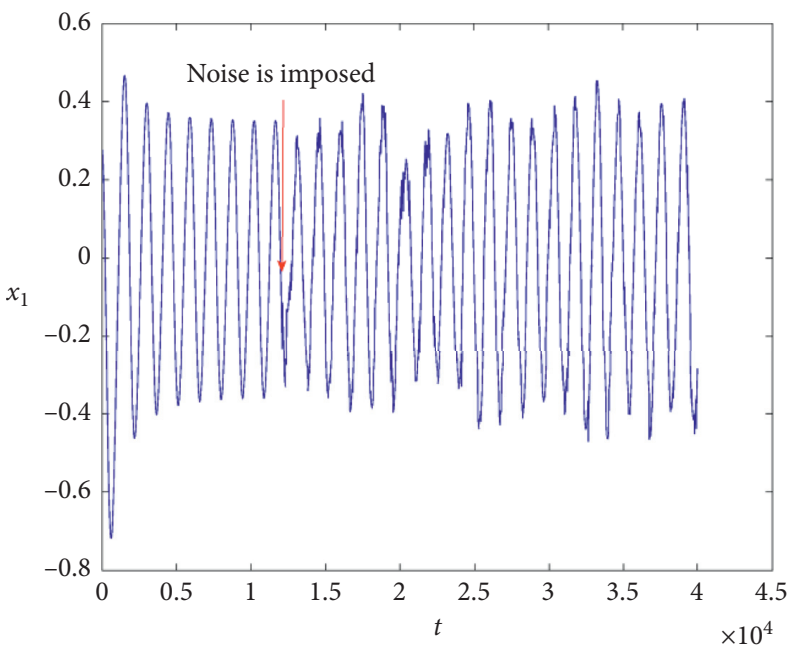

(a)

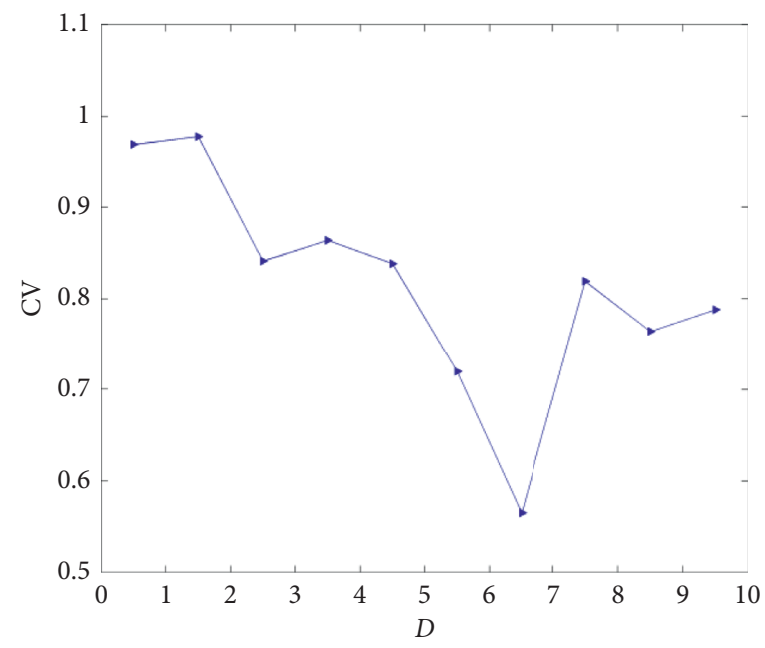

(b)

FIGURE 8: Time series of $x_{1}$ in FHNN (32) disturbed by noise and CV trend with noise intensity changing when parameters satisfy Theorem 3 in Case 2 for $\alpha=0.8$. (a) Time series of $x_{1}$; (b) CV trend with noise intensity $D$ changing.

\section{Conclusions}

Our main work is about the Mittag-Leffler stability and noise-induced coherence resonance of fraction-order Hopfield neuron network.

Firstly, stability of fraction-order Hopfield neuron network is analyzed and two criteria for judging whether fraction-order neuron network is globally stable are addressed. Numerical simulations are used to interpret the rationality and feasibility of the theoretical results. Furthermore, via analyzing the simulations, it can be obtained that system parameters have significant effect in describing neural network's dynamics. Particularly, an interesting phenomenon is found that, by selecting suitable system parameters, fraction-order Hopfield neuron network can stabilize to a limit cycle or two equilibriums accidently. The latter is a brand-new and important form of global stability and can be called global bistability.

Secondly, noise-induced coherence resonance of fraction-order Hopfield neural network is discussed. Numerical simulations indicate that, for given system parameters, whether the fractional-order Hopfield neural network tends to the equilibrium point or to an attractor domain, random noise can induce coherence resonance.

Results in this paper suggest that system parameters and random noise have significant impact on the dynamical behaviors of fraction-order Hopfield neuron network, which tells us that some potential work should be considered for fraction-order neuron network, like mechanisms underlying the bistability and the effect of system parameters on the 


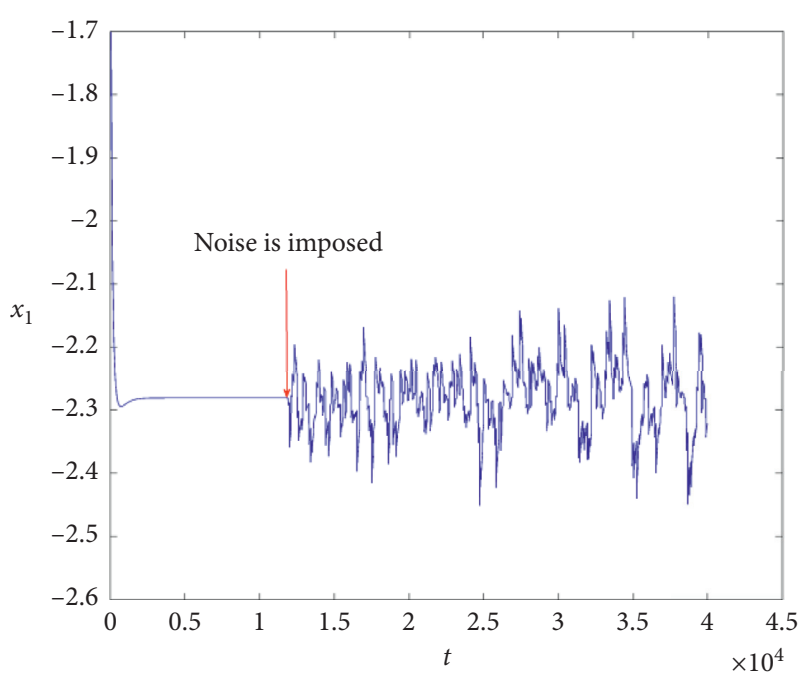

(a)

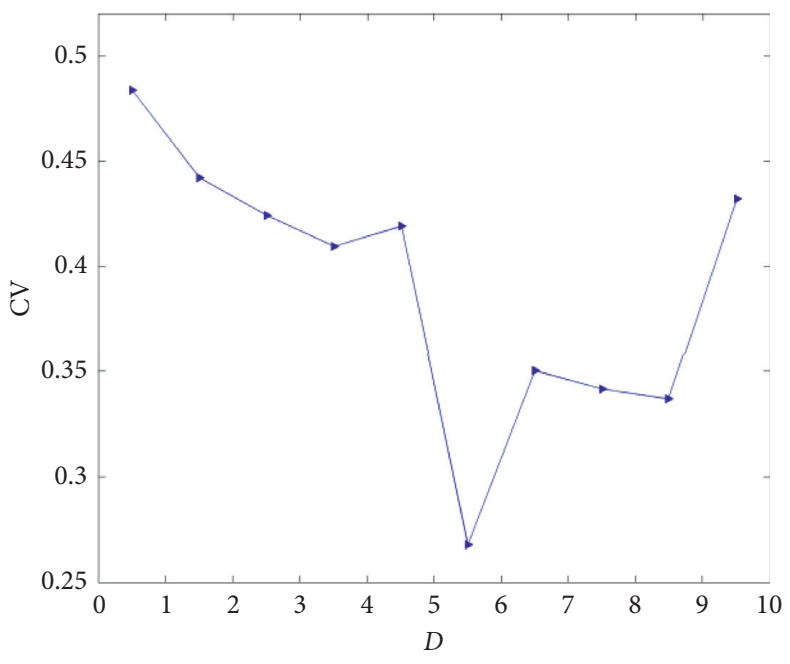

(b)

FIgURE 9: Time series of $x_{1}$ in FHNN (32) disturbed by noise and CV trend with noise intensity changing when parameters satisfy Theorem 3 in Case 3 for $\alpha=0.8$. (a) Time series of $x_{1}$; (b) CV trend with noise intensity $D$ changing.

coherence resonance. Furthermore, the dynamics of fraction-order neuron network with time delay and impulse coupling can be discussed along with time-delay-induced bistability or coherence resonance.

Dynamical behaviors of fraction-order neural network are of great significance due to its wide application. For example, the stability analysis of fraction-order neural network can provide theoretical basis for design of new circuits, such as multistable trigger and soft switch circuit. Noise-induced coherence resonance of fraction-order Hopfield neural network is helpful for understanding the dynamics of neuron system in noisy environment and diagnosing certain nervous system diseases.

\section{Data Availability}

The data used in our study are included within the article, and the authors used no other data. Researchers can verify the results of the article, replicate the analysis, and conduct secondary analyses using the data in the paper.

\section{Conflicts of Interest}

The authors declare that they have no conflicts of interest.

\section{Acknowledgments}

This work was supported by the National Natural Science Foundation of China (Grants nos. 11872327 and 51777180).

\section{References}

[1] Q. Lai, B. Norouzi, and F. Liu, "Dynamic analysis, circuit realization, control design and image encryption application of an extended Lü system with coexisting attractors," Chaos, Solitons \& Fractals, vol. 114, pp. 230-245, 2018.

[2] Q. Lai, A. Akgul, C. Li, G. Xu, and Ü. Çavuşoğlu, "A new chaotic system with multiple attractors: dynamic analysis, circuit realization and s-box design," Entropy, vol. 20, no. 1, p. 12, 2018.

[3] Q. Lai, P. D. K. Kuate, F. Liu, and H. H. C. Lu, "An extremely simple chaotic system with infinitely many coexisting attractors," IEEE Transactions on Circuits and Systems II: Express Briefs, vol. 66, no. 6, pp. 1129-1133, 2019.

[4] J. Ma, G. Zhang, T. Hayat, and G. Ren, "Model electrical activity of neuron under electric field," Nonlinear Dynamics, vol. 95, no. 2, pp. 1585-1598, 2019.

[5] M. Lv, J. Ma, Y. G. Yao, and F. Alzahrani, "Synchronization and wave propagation in neuronal network under field coupling," Science China-Technological Sciences, vol. 62, no. 3, pp. 448-457, 2019.

[6] Z. Yao, J. Ma, Y. Yao, and C. Wang, "Synchronization realization between two nonlinear circuits via an induction coil coupling," Nonlinear Dynamics, vol. 96, no. 1, pp. 205-217, 2019.

[7] Z. Liu, J. Ma, G. Zhang, and Y. Zhang, "Synchronization control between two Chua's circuits via capacitive coupling," Applied Mathematics and Computation, vol. 360, pp. 94-106, 2019.

[8] Z. T. Njitacke, I. S. Doubla, J. Kengne, and A. Cheukem, "Coexistence of firing patterns and its control in two neurons coupled through an asymmetric electrical synapse," Chaos, vol. 30, Article ID 023101, 2020.

[9] H. Bao, W. Liu, and A. Hu, "Coexisting multiple firing patterns in two adjacent neurons coupled by memristive electromagnetic induction," Nonlinear Dynamics, vol. 95, no. 1, pp. 43-56, 2019.

[10] P. Chittora, A. Singh, and M. Singh, "Application of Hopfield neural network for harmonic current estimation and shunt compensation," Electric Power Components and Systems, vol. 46, no. 3, pp. 290-301, 2018.

[11] J. Cao and T. Chen, "Globally exponentially robust stability and periodicity of delayed neural networks," Chaos, Solitons \& Fractals, vol. 22, no. 4, pp. 957-963, 2004.

[12] X. Sun, Z. Liu, and M. Perc, "Effects of coupling strength and network topology on signal detection in small-world neuronal networks," Nonlinear Dynamics, vol. 96, no. 3, pp. 2145-2155, 2019.

[13] Y.-W. Wang, W. Yang, J.-W. Xiao, and Z.-G. Zeng, "Impulsive multisynchronization of coupled multistable neural 
networks with time-varying delay," IEEE Transactions on Neural Networks and Learning Systems, vol. 28, no. 7, pp. 1560-1571, 2017.

[14] Q. Lai, B. Hu, Z.-H. Guan, T. Li, D.-F. Zheng, and Y.-H. Wu, "Multistability and bifurcation in a delayed neural network," Neurocomputing, vol. 207, pp. 785-792, 2016.

[15] J. J. Hopfield, "Neurons with graded response have collective computational properties like those of two-state neurons," Proceedings of the National Academy of Sciences, vol. 81, no. 10, pp. 3088-3092, 1984.

[16] B. Bao, H. Qian, J. Wang et al., "Numerical analyses and experimental validations of coexisting multiple attractors in Hopfield neural network," Nonlinear Dynamics, vol. 90, no. 4, pp. 2359-2369, 2017.

[17] B. Bao, H. Qian, Q. Xu, M. Chen, J. Wang, and Y. Yu, "Coexisting behaviors of asymmetric attractors in hyperbolictype memristor based Hopfield neural network," Frontiers in Computational Neuroscience, vol. 11, no. 81, pp. 1-14, 2017.

[18] Z. T. Njitacke and J. Kengne, "Complex dynamics of a 4D Hopfield neural networks (HNNs) with a nonlinear synaptic weight: coexistence of multiple attractors and remerging Feigenbaum trees," AEU-International Journal of Electronics and Communications, vol. 93, pp. 242-252, 2018.

[19] Z. T. Njitacke, J. Kengne, and H. B. Fotsin, "Coexistence of multiple stable states and bursting oscillations in a $4 \mathrm{D}$ Hopfield neural network," Circuits, Systems, and Signal Processing, vol. 39, no. 7, pp. 3424-3444, 2020.

[20] F. Meng, X. Zeng, and Z. Wang, "Impulsive anti-synchronization control for fractional-order chaotic circuit with memristor," Indian Journal of Physics, vol. 93, no. 9, pp. 1187-1194, 2019.

[21] G. Cottone, M. D. Paola, and R. Santoro, "A novel exact representation of stationary colored Gaussian processes (fractional differential approach)," Journal of Physics A: Mathematical and Theoretical, vol. 43, no. 8, Article ID 085002, 2010.

[22] F. Mainardi, "Fractional relaxation-oscillation and fractional diffusion-wave phenomena," Chaos, Solitons \& Fractals, vol. 7, no. 9, pp. 1461-1477, 1996.

[23] C. J. Xu, M. X. Liao, P. L. Li, Q. M. Xiao, and S. Yuan, "PD9 control strategy for a fractional-order chaotic financial model," Complexity, vol. 2019, Article ID 2989204, 14 pages, 2019.

[24] M. Xiao, W. X. Zheng, J. Lin, G. Jiang, L. Zhao, and J. Cao, "Fractional-order PD control at Hopf bifurcations in delayed fractional-order small-world networks," Journal of the Franklin Institute, vol. 354, no. 17, pp. 7643-7667, 2017.

[25] C. Xu, M. Liao, P. Li, Y. Guo, Q. Xiao, and S. Yuan, "Influence of multiple time delays on bifurcation of fractional-order neural networks," Applied Mathematics and Computation, vol. 361, pp. 565-582, 2019.

[26] C. Xu, P. Li, M. Liao, and S. Yuan, "Bifurcation analysis for a fractional-order chemotherapy model with two different delays," Mathematical Methods in the Applied Sciences, vol. 43, no. 3, pp. 1053-1083, 2020.

[27] C. Huang, J. Cao, M. Xiao, A. Alsaedi, and T. Hayat, "Effects of time delays on stability and Hopf bifurcation in a fractional ring-structured network with arbitrary neurons," Communications in Nonlinear Science and Numerical Simulation, vol. 57, pp. 1-13, 2018.

[28] C. J. Xu, P. L. Li, M. X. Liao, Z. X. Liu, Q. M. Xiao, and S. Yuan, "Control scheme for a fractional-order chaotic Genesio-Tesi model," Complexity, vol. 2019, Article ID 4678394, 15 pages, 2019.
[29] Z. Wang, Y. Xie, J. Lu, and Y. Li, "Stability and bifurcation of a delayed generalized fractional-order prey-predator model with interspecific competition," Applied Mathematics and Computation, vol. 347, pp. 360-369, 2019.

[30] C. Xu, M. Liao, and P. Li, "Bifurcation of a fractional-order delayed malware propagation model in social networks," Discrete Dynamics in Nature and Society, vol. 2019, Article ID 7057052, 10 pages, 2019.

[31] C. Xu, M. Liao, P. Li, and J. Yan, "Influence of time delay on bifurcation in fractional order BAM neural networks with four delays," IEEE Access, vol. 7, no. 1, pp. 70955-70965, 2019.

[32] A. S. Deshpande, V. Daftardar-Gejji, and Y. V. Sukale, "On Hopf bifurcation in fractional dynamical systems," Chaos, Solitons \& Fractals, vol. 98, pp. 189-198, 2017.

[33] J. Yu, C. Hu, and H. Jiang, " $\alpha$-stability and $\alpha$-synchronization for fractional-order neural networks," Neural Networks: The Official Journal of the International Neural Network Society, vol. 35, pp. 82-87, 2012.

[34] I. Stamova, "Global Mittag-Leffler stability and synchronization of impulsive fractional-order neural networks with time-varying delays," Nonlinear Dynamics, vol. 77, no. 4, pp. 1251-1260, 2014.

[35] H. Wang, Y. Yu, G. Wen, S. Zhang, and J. Yu, "Global stability analysis of fractional-order Hopfield neural networks with time delay," Neurocomputing, vol. 154, pp. 15-23, 2015.

[36] H. Wu, X. Zhang, S. Xue, L. Wang, and Y. Wang, "LMI conditions to global Mittag-Leffler stability of fractional-order neural networks with impulses," Neurocomputing, vol. 193, pp. 148-154, 2016.

[37] P. Liu, X. Nie, J. Liang, and J. Cao, "Multiple Mittag-Leffler stability of fractional-order competitive neural networks with Gaussian activation functions," Neural Networks, vol. 108, pp. 452-465, 2018.

[38] I. Podlubny, Fractional Differential Equations: An Introduction to Fractional Derivatives, Fractional Differential Equations, to Methods of Their Solution and Some of Their Applications, Academic Press, Cambridge, MA, USA, 1st edition, 1998.

[39] A. A. Kilbas, H. M. Srivastava, and J. J. Trujillo, Theory and Applications of Fractional Differential Equations, Elsevier Science Limited, Amsterdam, Netherlands, 2006.

[40] Y. Li, Y. Chen, and I. Podlubny, "Mittag-Leffler stability of fractional order nonlinear dynamic systems," Automatica, vol. 45, no. 8, pp. 1965-1969, 2009.

[41] Y. Li, Y. Chen, and I. Podlubny, "Stability of fractional-order nonlinear dynamic systems: Lyapunov direct method and generalized Mittag-Leffler stability," Computers \& Mathematics with Applications, vol. 59, no. 5, pp. 1810-1821, 2010.

[42] J. Chen, Z. Zeng, and P. Jiang, "Global Mittag-Leffler stability and synchronization of memristor-based fractional-order neural networks," Neural Networks, vol. 51, pp. 1-8, 2014.

[43] K. Diethelm, N. J. Ford, and A. D. Freed, "A predictor-corrector approach for the numerical solution of fractional differential equations," Nonlinear Dynamics, vol. 29, no. 1, pp. 3-22, 2002.

[44] C. Chen, H. Bao, M. Chen, Q. Xu, and B. Bao, "Non-ideal memristor synapse-coupled bi-neuron Hopfield neural network: numerical simulations and breadboard experiments," AEU-International Journal of Electronics and Communications, vol. 111, Article ID 152894, 2019.

[45] C. Chen, J. Chen, H. Bao, M. Chen, and B. Bao, "Coexisting multi-stable patterns in memristor synapse-coupled Hopfield neural network with two neurons," Nonlinear Dynamics, vol. 95, no. 4, pp. 3385-3399, 2019. 